\title{
A randomized controlled trial on effect of transcranial direct current stimulation and cognitive behavioral therapy on headache related disability and impact of headache on life of migraineurs
}

\author{
Manoj Malik', Shailendra Kumar Singh ${ }^{1}$, Narkeesh Arumugam² \\ ${ }^{1}$ Department of Physiotherapy, Guru Jambheshwar University of Science and Technology, \\ Hisar, Haryana, India \\ ${ }^{2}$ Department of Physiotherapy, Punjabi University, Patiala, India
}

\begin{abstract}
Introduction. Migraine is a public health problem of great impact on patients as well as society. Migraine prophylaxis requires daily administration of anti-migraine compounds whether or not migraine attack is occurring. All the drugs used for migraine prevention have potential and often relevant adverse effect or contraindications. The purpose of present study was to evaluate efficacy of non-pharmacological management techniques like Cognitive Behavioral Therapy (CBT) and Transcranial Direct Current Stimulation (TDCS) on headache related disability and impact of headaches on life of patients suffering from migraine.

Methodology. Sixty six subjects fulfilling the selection criteria were recruited for the study. They were randomly allocated into three groups. Group one received TDCS, Group 2 received CBT, and Group 3 (Control group) did not receive any treatment. Participants were asked to fill HDI and HIT 6 prior to start of intervention, post intervention and at follow up. Collected data was analyzed for statistical significance.

Results. We found a significant decrease in HDI and HIT 6 scores in TDCS and CBT Group as compared to Control Group. This improvement was maintained during follow up period.

Conclusion. TDCS and CBT can be effective in decreasing headache related disability and impact of headache on daily life in patients suffering from Migraine.
\end{abstract}

Keywords: migraine, cognitive behavioral therapy, transcranial direct current stimulation

\begin{abstract}
Abbreviations
CBT - Cognitive Behavioural Therapy

TDCS - Transcranial Direct Current Stimulation

HDI - Headache Disabilty Index

HIT 6 - Headache Impact Test 6)
\end{abstract}

\section{INTRODUCTION}

Migraine is the most common headache diagnosis reported to neurological services in Asia. However, Migraine remains under diagnosed and under treated in this region even though there is a high disability in patient with migraine (1). Disability, defined as any restriction and lack of ability to perform an activity in the manner or in the range con- sidered normal for a human being is a useful measure for assessing the impact of migraine on patient's health and functioning (2). Migraine is a public health problem with great impact on patients as well as society. The annual cost of migraine related lost productivity is enormous. Migraine attacks are usually characterized as unilateral, pulsating, severe headache, lasting from four to seventy two 
hours, often accompanied with nausea, vomiting, phonophobia and photophobia (3). The WHO consider a day lived with severe migraine as a disabling as a day lived with dementia, quadriplegia, angina or rheumatoid arthritis. Improving our understanding of migraine and taking steps to reduce headache related disability have been identified as a urgent public health priority by WHO in global campaign to lift the burden of migraine (4). Cortical spreading depression (CSD), discovered by laeo in 1944, had been implicated in pathogenesis of migraine. CSD is characterized by a slowly spreading wave of neuronal and glial depolarization lasting for about one minute that develops within the brain area such as cerebral cortex, cerebellum or hippocampus after electrical or chemical stimulation (5). Pathogenesis of migraine is related to an imbalance in activity between brain stem nuclei regulating anti-nociception and vascular control. Experimental and clinical data suggest a temporary aseptic perivascular inflammation in the dura from trigeminal antidromic release of neuropeptides such as calcitonin gene related peptide, substance $\mathrm{P}$ and other tachykinins (6). Trigeminal nerve innervate the meninges of brain and trigeminal vascular afferents get triggered by CSD, thus provoking the cortical meningeal and brain stem region and it is followed by development of headache (7). Migraine prophylaxis requires daily administration of anti-migraine compounds whether or not migraine attack is occurring. All the drugs used for migraine prevention have potential and often relevant adverse effect or contraindications, and may also interfere with other concurrent conditions and treatments. These problems may result in poor patient's compliance towards prophylactic pharmacological management of Migraine. A number of alternate treatment methods like manual therapy, neuromodulatory techniques, biofeedback, dietary changes and behavioural therapy approaches have been extensively studied for their efficacy in migraine prophylaxis (8). Strong electrical currents have been delivered to the patients for the relief of headache and epilepsy since ancient times. Scribonuislargus, Pliny the elder and Galen, all had reportedly used Torpedo electric fish to elicit a sudden transient stupor. The effect of electrical stimulation were subsequently studied using modern scientific methods by many scientist notably
Galvani and Volta (9). There is increasing interest in the therapeutic and investigative capabilities of noninvasive forms of electrical brain stimulation such as Transcranial Direct Current Stimulation(TDCS). TDCS is a noninvasive brain stimulation technique that applies in mild (1-2mA) direct electrical current through scalp electrodes (10). Transcranial application of weak direct current induces intracerebral current flow which alters neuronal activity and behavior. TDCS modulates human cerebral cortical function and induces focal, prolonged and reversible shift of cortical excitability (11).

Cognitive behavioural therapy is a (CBT) is a time limited skill based therapy which requires active participation of patient. Therapist and patient work together to enhance the subject's understanding, insights and use of cognitive behavior strategies so that the he will continue to use CBT technique even after the end of therapy (12). CBT is one of the most extensively applied technique in the field of psychotherapy. A number of studies have been done to evaluate the efficacy of CBT in conditions like depression, anxiety, schizophrenia etc. The purpose of present study was to evaluate the effect of CBT and TDCS on headache related disability and impact of headaches on life of patients suffering from Migraine.

\section{METHODOLOGY}

\section{Study Design, Ethical Approval and Selection Criteria}

The design of the trial was randomized controlled interventional study. The protocol was assessed and approved by Institution Ethics Committee (IEC) of Department of Physiotherapy, Guru Jambheshwar University of Science and Technology, Hisar vide letter no. PTY/2015/800, dated $3 / 11 / 15$. Constitution of IEC of Department is in accordance with the guidelines of Indian Council of Medical Research. Sixty six Volunteers suffering from Migraine were enrolled for the study (47 Females and 19 Males) after screening for selection criteria. Patients were eligible for the study if they fulfilled the criteria for migraine as defined by Headache classification Committee of International Headache Society, International classification of Headache Disorders, 3rdEdition (13). Patients of any gender between 18 to 65 years of age, not tak- 
ing any prophylactic medication or any herbal hair oil massage were included in the study. Subjects with any co-existent medical and psychiatric disorder as well as any metallic implant in head, skull defect or pacemaker were excluded.

\section{Intervention}

A baseline screening was done for selection criteria. Subjects fulfilling selection criteria, were randomly allocated to either the experimental groups (Group 1 received TDCS, Group 2 received CBT) or in control group. Randomization was done using computer generated random number tables. Written informed consent was obtained prior to commence- ment of study. Participants were asked to fill Headache disability index (HDI) and Headache impact test 6(HIT 6) score prior to intervention, post intervention and at the time of follow up after one month post intervention. Subjects in Group 1 were given TDCS, three sessions per week for six weeks duration, cathode over $\mathrm{Oz}$ and anode over $\mathrm{Cz}$ electrode positions according to 10-20 EEG system. A constant current of $2 \mathrm{~mA}$ for 20 minutes was given to every subject in Group 1. Subjects in group 2 were given CBT sessions, one session per week, of approximately one hour duration for six weeks along with Sham TDCS, for Sham stimulation electrodes were placed in the same position as the TDCS

\section{CONSORT diagram showing the flow of participants through each} stage of randomized controlled trial
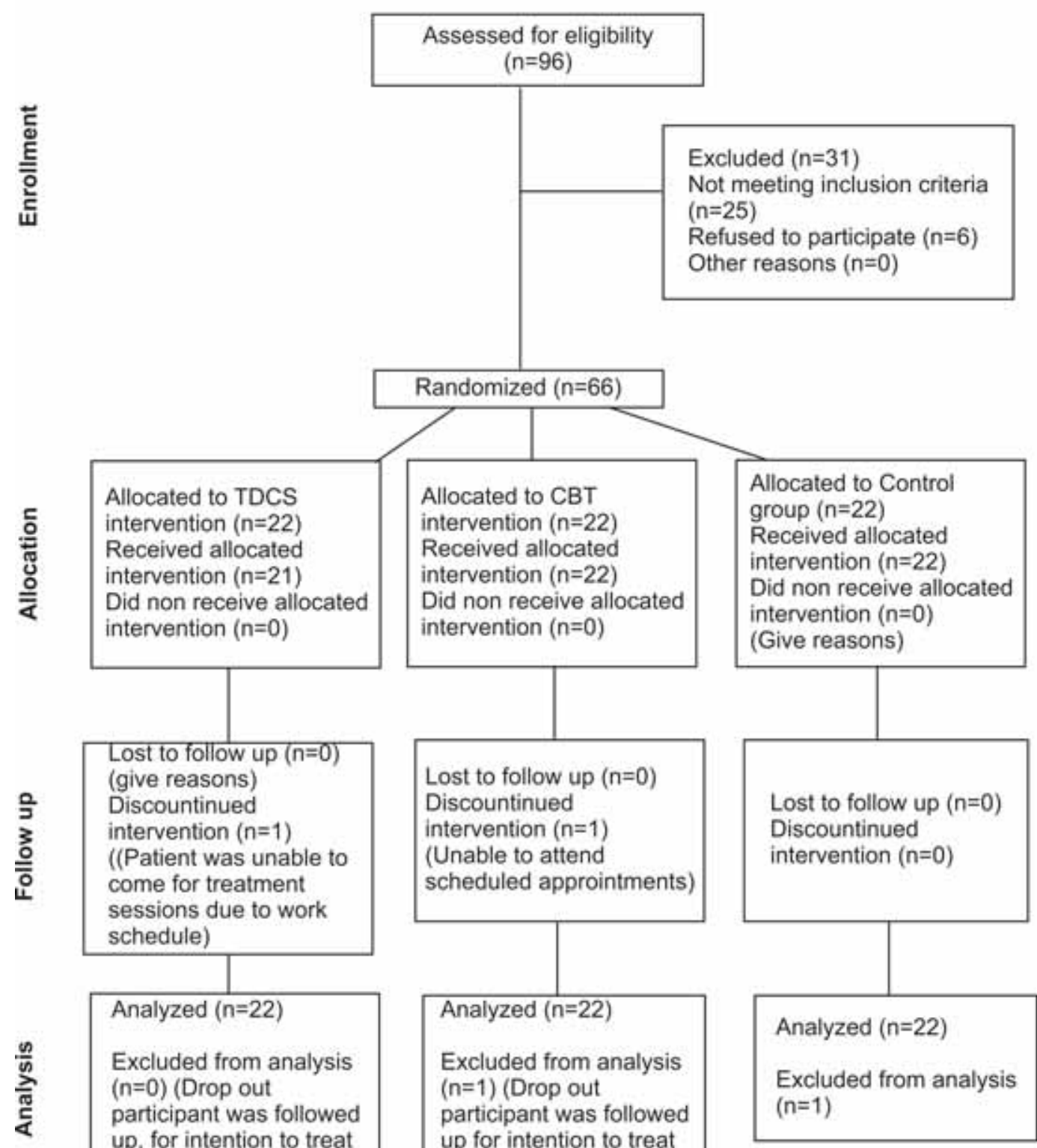
up, for intention to treat analysis) 
Group. Stimulation of $2 \mathrm{~mA}$ was given for thirty seconds, after 30 seconds the intensity was ramped off slowly up to zero. Sham TDCS was given for twenty minutes. The control group did not receive any treatment. Only pre intervention, post intervention and follow up data was collected from the subjects in control group. All the data obtained was analyzed for statistical significance. The Consolidated standards of Reporting Trials (CONSORT) flow chart describing the study progress through different phases of trial is displayed in Fig. 1.

\section{DATA ANALYSIS AND RESULTS}

Sample Size Calculation-A sample size calculation was performed using the following parameters $(\alpha=0.05$, power of 0.8$)$, considering a drop our rate of $10 \%$, and minimal clinically important change of eight points of HIT 6 Questionnaire (14).

\section{Data Analysis}

The Normality of collected data was analyzed by Shapiro-wilk Test. The Outcome measures were normally distributed. Therefore, parametric tests were applied for analysis. Descriptive data was expressed in terms of mean and standard deviation and compared with one way ANOVA and Post Hoc analysis using Tukey test for statistical significance both within the group as well as among groups. Significance level was set at $\mathrm{P} \leq 0.05$. All the analysis was based on intention to treat principle. Two subjects dropped out from the study (one in CBT and one in TDCS). They were followed up and data was obtained from them. Statistical analysis was performed using Statistical Package for the Social Sciences, 16 version for Window.

\section{Baseline Comparisons}

Sixty six subjects (47 females and 19 males) participated in the study. Mean age of participants was $33.80 \pm 11.50$. Baseline scores of HDI and HIT 6 were similar in all three groups. Table 1 and Fig. 2 depict the baseline data characteristics.

TABLE 1. Showing baseline mean HDI and HIT 6 score of all the groups with $P$ value of comparison among groups at pre intervention level

\begin{tabular}{|l|c|c|c|c|}
\hline & $\begin{array}{c}\text { CBT } \\
\text { Mean } \pm \text { SD }\end{array}$ & $\begin{array}{c}\text { TDCS } \\
\text { Mean } \pm \text { SD }\end{array}$ & $\begin{array}{c}\text { CONTROL } \\
\text { Mean } \pm \text { SD }\end{array}$ & P Value \\
\hline HDI & $64.18 \pm 14.30$ & $59.09 \pm 13.40$ & $57.27 \pm 14.66$ & .251 \\
\hline HIT 6 & $67.36 \pm 5.18$ & $66.23 \pm 2.95$ & $64.36 \pm 4.52$ & .075 \\
\hline
\end{tabular}

Results suggest that Pre Intervention scores of all the three groups were similar.

\section{HDI Comparisons}

There was significant difference among pre intervention, post intervention and follow up scores in TDCS and CBT group. Post hoc analysis showed significant improvement in HDI scores at post intervention and follow up when compared to pre intervention in both CBT and TDCS group while there was no significant difference in post intervention and follow up in TDCS and CBT Group. However, there was no significant difference among pre intervention, post intervention and follow up scores in control group.

Table 2 summarizes the means and standard deviation of HDI scores of all groups at different levels and their significance levels.

Table 2 and Fig. 3 shows HDI mean scores and standard deviation at different points of protocol.

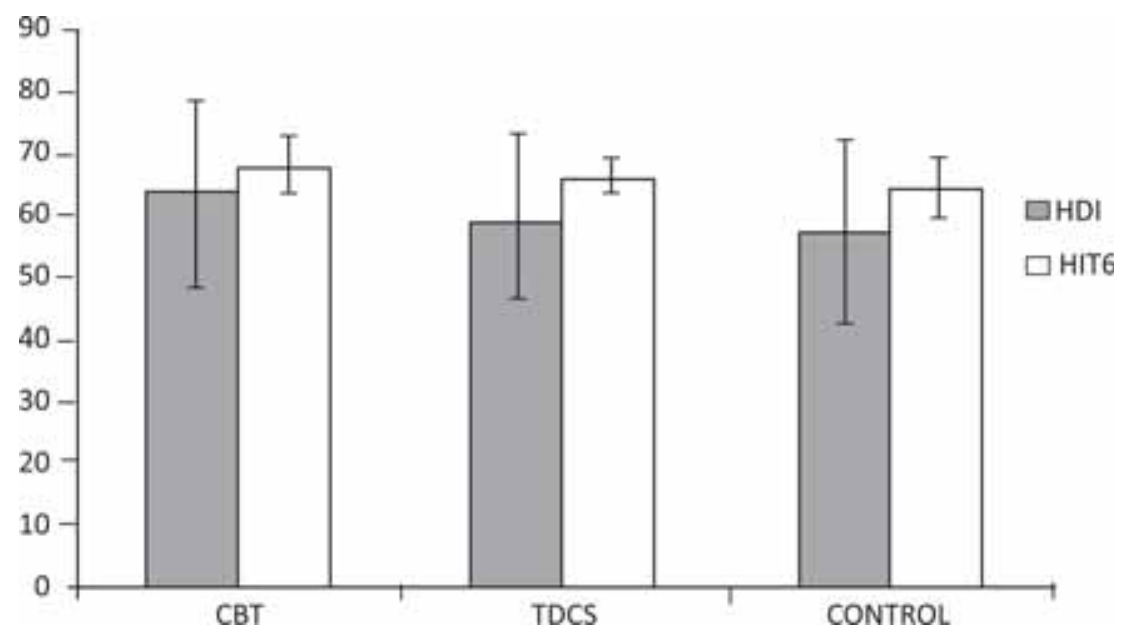

FIGURE 2. Depicts Mean HDI and HIT6 Scores and standard deviation at Preintervention level 
TABLE 2. Showing mean HDI score of all the groups at different points of Protocol and their statistical significance

\begin{tabular}{|l|c|c|c|c|}
\hline Groups & $\begin{array}{c}\text { Baseline } \\
\text { Mean } \pm \text { SD }\end{array}$ & $\begin{array}{c}\text { Post } \\
\text { intervention } \\
\text { Mean } \pm \text { SD }\end{array}$ & $\begin{array}{c}\text { Follow up } \\
\text { Mean } \pm \text { SD }\end{array}$ & P value \\
\hline CBT & $64.18 \pm 14.30$ & $34.72 \pm 12.74$ & $36.00 \pm 11.88$ & .0001 \\
\hline TDCS & $59.09 \pm 13.45$ & $29.27 \pm 7.44$ & $32.27 \pm 7.93$ & .0001 \\
\hline CONTROL & $57.27 \pm 14.66$ & $56.90 \pm 15.17$ & $57.00 \pm 14.95$ & .996 \\
\hline
\end{tabular}

There was significant difference in comparisons among groups for mean change in HDI scores from baseline to post intervention levels .Post Hoc analysis showed a significant decrease in HDI scores of TDCS $(29.81 \pm 8.96)$ and CBT $(29.45 \pm 10.01)$ group as compared to control group (36 \pm 3.7$)$. However there was no significant difference between CBT and TDCS group. Result suggests improvement in HDI scores of migraineurs in CBT and TDCS group and this improvement was maintained in follow up.

\section{HIT 6 Comparisons}

There was significant difference among pre intervention, post intervention and follow up scores in TDCS and CBT group. Post hoc analysis showed significant decrease in HIT 6 scores at post intervention and follow up when compared to baseline in both CBT and TDCS group while there was no significant difference in post intervention and follow up in TDCS and CBT Group. However, there was no significant difference among baseline, post intervention and follow up scores in control group. Table 3 summarizes the means, standard deviation and statistical significance of HIT 6 scores of all groups at different levels. Table 3 and Fig. 4 shows mean HIT 6 scores and Standard Deviation at different points of protocol.

TABLE 3. Showing HIT 6 score of all the groups at different levels of protocol and their statistical significance

\begin{tabular}{|l|c|c|c|c|}
\hline Groups & $\begin{array}{c}\text { Baseline } \\
\text { Mean } \pm S D\end{array}$ & $\begin{array}{c}\text { Post } \\
\text { Intervention } \\
\text { Mean } \pm S D\end{array}$ & $\begin{array}{c}\text { Follow up } \\
\text { Mean } \pm S D\end{array}$ & P value \\
\hline CBT & $67.36 \pm 5.18$ & $56.45 \pm 5.91$ & $55.46 \pm 5.58$ & .0001 \\
\hline TDCS & $66.23 \pm 2.95$ & $55.18 \pm 3.15$ & $56.27 \pm 3.38$ & .0001 \\
\hline CONTROL & $64.36 \pm 4.52$ & $64.45 \pm 3.20$ & $63.77 \pm 3.10$ & .800 \\
\hline
\end{tabular}

There was significant difference in comparisons among groups for mean change in HIT 6 scores from baseline to post intervention levels. Post Hoc analysis shows a significant decrease in HIT 6 scores of TDCS $(11.05 \pm 3.48)$ and CBT $(10.91 \pm 4.58)$ group as compared to control group $(-0.09 \pm 2.44)$. However there was no significant difference between CBT and TDCS group. Result suggests significant decrease in HIT 6 scores of migraineurs in CBT and TDCS group and this improvement was further maintained in follow up period indicating the success of therapy.

\section{Discussion}

Results of present study suggest that TDCS and CBT have significant effect in reducing headache related disability and its impact on various domains of patient's life. It is recognized that burden of episodic headaches on patients is multidimensional. HIT6 scale is a global measure of the impact of headache and it is correlated with clinical parame-

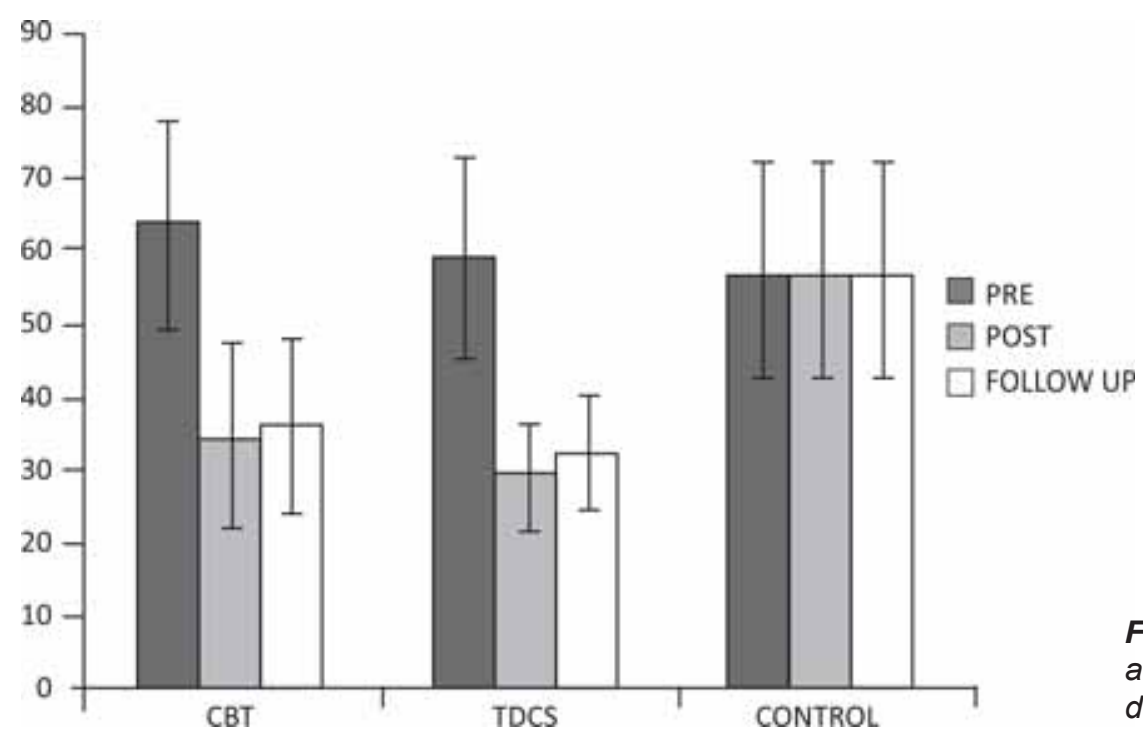

FIGURE 3. Depicts the mean HDI Scores and standard deviation of the groups at different points of protocol 


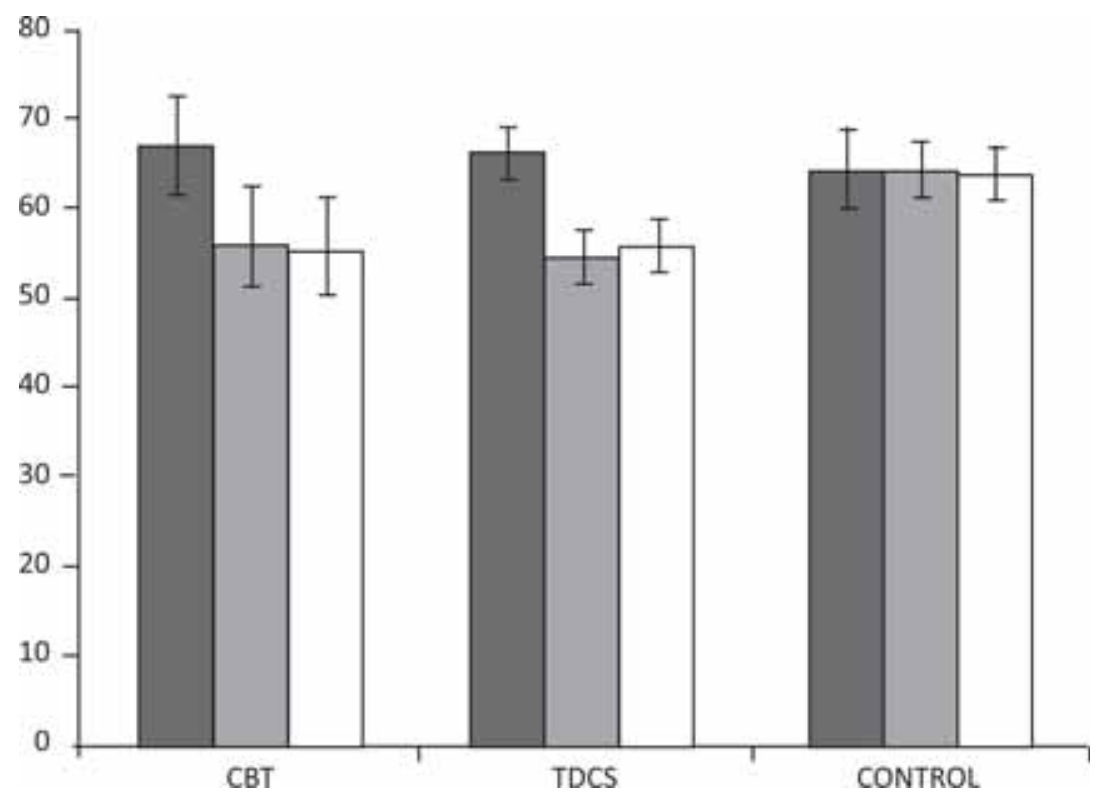

FIGURE 4. Depicts mean HIT Scores and standard deviation at different points of protocol ters of headache such as severity as well as with patient related outcomes such as quality of life across different diagnostic groups of headaches (15). The headache disability inventory is also a convenient scale to asses headache related affective distress. It is designed to assess the impact of headaches on daily functioning and daily activity (16). Therefore, these two scales are reliable measures to assess the treatment effect on disability and daily life of patients suffering from migraine. There is a strong correlation between HIT6 scores and duration of disability recorded in headaches diaries. HIT6 scores are closely related to overall burden of migraine and is useful for assessing headache related disability over one month period (17). Primary headache may have an extensive impact and significant negative consequences on patient's life. Migraine has a pervasive effect on daily functioning with overall level of functional disability higher in chronic headache sufferers (18).

Migraine is associated with high levels of headache related disability and high levels of headache related disability reflects unmet treatment needs. Onset of migraine headaches and treatment efficacy is unpredictable. This along with the disruption of lives in migraine patient produce an ongoing uncertainly and disequilibrium. This framework includes factors such as personal factors and illness related factor, which may influence the person's cognitive appraisal and selection of coping strategies. Depressive symptomatology is depicted as affecting migraine headache, chronic pain experi- ence and coping with disability. (19)CBT can be an effective approach in decreasing headache related disability and improving quality of life in migraine patients. One of the possible factors that correlate with treatment effect of CBT in migraineurs is the association between migraine and major depression. Major depression increases the risk of migraine and migraine increases the risk of major depression. This bidirectional association is not observed in other severe headaches (20). Further, attachments style, an enduring psychological tract influence disability levels in migraine patients. Attachment style was significantly associated with symptoms with unsecure patients having a significantly greater number of physical symptoms compared to secure patients. Comorbid depressive symptoms also play role in modulating the impact of migraine on every day functioning (3). Previous research suggests that spiritual meditation may ameliorate some of negative traits associated with migraine headaches, corroborating the association of migraine headaches with depression and anxiety. Spiritual meditation results in decrease in frequency of migraine headache, anxiety and negative effect, increase in pain tolerance, headache related self-efficacy, daily spiritual experiences and existential wellbeing (21). CBT is an effective approach in management of recurrent depression (22), comorbid major depression with insomnia (23) and depression in adolescents (24). CBT may influence clinical recovery in major depression by modulating the functioning of specific sites in limbic and 
cortical region (25). The association of major depression with migraine and efficacy of CBT in depression may explain the efficacy of CBT in decreasing disability and improving quality of life, parameters in migraine patients. Migraine can be triggered by psychological stressors; severe migraine can itself cause significant psychological stress, which in turn exacerbates the problem. Even if psychological stress is not significantly involved in the genesis of headache, pain management technique can help cope up with the pain more effectively (26).

Results of the present study suggest that decrease in headache related disability and impact of headache on daily life with TDCS stimulation on $\mathrm{Cz}-\mathrm{Oz}$ montage placement. We placed cathode over $\mathrm{Oz}$ and anode over Cz. Alexandra F. Dasilva et al. did a study on neuroanatomical target analysis of high definition and conventional TDCS montages used for migraine and pain control. They concluded that this montage results in current flow mainly to parietal and occipital lobes with maximum electrical field occurring at primary visual cortex (27). A generalized cortical interictal hyper excitability, more pronounced in visual cortex has been suggested in migraine (28). Occipital cortex is hyper excitable in the migraine interictum, both in migraine with and without aura (29). The evaluation of motor and occipital cortex excitability in migraine patients using transcranial magnetic stimulation resulted in finding that suggested hyperexcitability in occipital cortex rather than the motor cortex (30). There is strong evidence that neurons underlying the cathode in TDCS are inhibited with resting membrane potential shifting towards hyperpolarization and reduce neuronal firing (9). Therefore, it may be suggested that cathodal TDCS at Oz may result in decrease excitation at occipital cortex, thus affecting the migraine pathophysiology and resulted in significant reduction in HDI and HIT 6 scores showing improvement in migraneurs.

Migraine management is a difficult task and decreasing disability related to the disease and its negative impact on patient's life can be challenging. TDCS and CBT can offer a hope to these patients. One of the positive aspects of these interventions is that they are free from any significant adverse effect. Future studies to evaluate the efficacy of TDCS combined with CBT in migraine management as well as effect of non-invasive other neurostimulation techniques combined with behavioral therapy can be studied on recovery outcomes of migraine. The present study has some limitations and their discussion is also warranted. Authors could not investigate the neural excitability changes after TDCS and CBT. Neuroimaging prior to and after treatment protocol could have provided significant insights to the pathophysiological changes . Further, our study group was limited to population of particular area. In spite of this limitation our study has suggested positive outcomes of TDCS and CBT in migraine management. Therefore, further studies can be done on bigger and more heterogeneous sample population.

\section{CONCLUSION}

We concluded that TDCS and CBT are effective in decreasing headache related disability and the impact of migraine headaches on life of patients. There was no significant difference between genders, age group and duration of disease in efficacy of TDCS and CBT. These techniques hold a lot of potential on effective management of quality of life of migraineurs. Future researches can be done to more effectively utilize the potential of these techniques in migraine management.

\section{Acknowledgement}

The Authors would like to thank Professor Ved Pal for his insightful suggestion regarding Data Analysis.

\section{Conflict of Interest}

There is no conflict of interest.

\section{Funding}

This study is not funded by any Funding Agency. 


\section{REFERENCES}

1. Wang S.J., Chung C.S., Chankrachang S. et al. Migraine disability Awareness campaign in Asia: Migraine Assessment for prophylaxis. Headache 2008; 48:1356-1365.

2. Rossi P., Lorenzo G., Malpezzi M.G., Lorenzo C.D. et al. Depressive symptoms And Insecure Attachments as Predictors of Disability in a Clinical Population of Patients With Episodic and Chronic Migraine. Headache 2005; 45:561-570.

3. Andreou A.P., Akerman S., Holland P.R. Neurobiology of Migraine. Neuroscience 2003; 4:386-398.

4. Blumenfeld A.M., Varon S.F., Wilcox T.K. et al. Disability ,HRQoL and resource use among chronic and episodic migraineurs:Results from the International Burden of Migraine Study (IBMS). Cephalagia 2011; 31:301-315.

5. Ayata C., Jin H., Kudo C. et al. Suppression of Cortical Spreading depression in Migraine prophylaxix. Ann Neurol 2006; 59:652-661.

6. Weiller C., May A., Limmroth V., Joptner M. et al. Brain stem activation in spontaneous human migraine attacks. Nat Med 1995; 1:658-660.

7. Girotra P., Singh S.K., Saini D. Disentangling the Intricacies of Migraine: A Review. CNS Neurol Disord Drugs targets, 2014; 13, 000-000:1-16.

8. Malik M., Singh S.K., Arumugam N.A. Case Study onEfficacy Of Transcranial Direct Current Stimulation In Management of Migraine. Int J Adv Res 2016; 4(11):972-975.

9. Stagg C.J., Nitsche M.A. Physiological Basis of Transcranial Direct Current Stimulation. Neuroscientist 2011; 17:37-53.

10. Arul-Anandam A.P., Loo C., Sachdev P. Transcranial direct current stimulation- What is the evidence for its efficacy and safety? F 1000 Med Rep 2009; 1:1-4.

11. Nitsche M.A., Cohen L.G., Wassermann E.M., Priori et al.Transcranial direct current stimulation: State of Art 2008. Brain Stimul 2008; 1:206-23.

12. Simmons J., Griffiths R. Structure of Therapy and Sessions.CBT for Beginners. Trefgarne S ( $2^{\text {nd }}$ Edition 2014), Great Britan: SAGE Publications Ltd; 34-45.

13. Headache Classification Committee of the International Headache Society. International classification of headache disorder 3rd edition (Beta Version). Cephalgia 2013; 33; 629-808.

14. Castien R.F., Blankenstein A.H., Windt D.A. et al. Minimal clinically important change on the Headache Impact Test-6 questionnaire in patients with chronic tension-type headache. Cephalalgia. 2012; 32:710-714.

15. Nachit-Ouinekh F., Dartigues J.F., Henry P. et al. Use of the headache impact test(HIT-6) in general practice: relationship with quality of life and severity. Eur J Neurol 2005; 12:189-193.
16. Holroyd K.A., Malinoski P., Davis M.K. et al. The three dimensions of headache impact: Pain, disability, and affective distress.Pain 1999; 83:571-578

17. Shin H.E., Park J.W., Kim Y. et al. Headache Impact Test-6(HIT-6) scores for migraine patients: Their Relation to disability as measured from a headache Diary. J Clin Neurol 2008; 4:158-163.

18. Bussone G., Usai S., Grazzi L. et al. Disability and quality of life in different primary headaches: results from Italian studies, Neurol Sci 2004; 25:S105-S107.

19. Kotoylo C.J., Broome M.E. Predicting Disability and Quality of Life in a Community Based Sample of Women With Migraine Headache. Pain Manag Nurs 2000; 1(4):139-151.

20. Breslau N., Lipton R., Schultz L., et al. Comorbidity of Migraine and Depression: Investigating potential etiology and prognosis. Neurology 2003; 60:1308-1312.

21. Wachholtz A.B., Pargament K.I. Migraines and meditation:Does spirituality matter? J Behav Med 2008; 31:351-366.

22. Fava G.A., Rafanelli C., Grandi S. et al. Prevention of Recurrent Depression With Cognitive behavioral Therapy. Arch Gen Psychiatry 1998; 55:816-820.

23. Manber R., Edinger J.D., Gress J.L. et al. Cognitive behavioral Therapy for Insomnia Enhances Depression Outcomes in Patients With Comorbid Major Depressive Disorder and Insomnia. Sleep 2008; 31: 489-495.

24. Reynold W.M., Coats K.I. A Comparison of Cognitive- behavioral Therapy and Relaxation Training for the Treatment of Depression in Adolescents. J Consult Clin Psychol 1986; 54(5):653-660

25. Goldapple K., Segal Z., Garson C. et al. Modulation of CorticalLimbic Pathways in Major Depression. Arch Gen Psychiatry 2006; 61:34-41.

26. Brown H., Newman C., Noad R. et al. Behavioral management of Migraine. Ann Indian Acad Neurol 2012; 15:78-82.

27. DaSilva A.F., Troung D.Q., DosSantos M.F. et al. State- of- art neuroanatomical target analysis of high definition and conventional tDCS montages used for migraine and pain control. Front Neuroanat 2015; 9:1-12

28. Brigo F., Storti M., Tezzon F. et al. Primary visual Cortex Excitability in migraine: a systematic review with meta - analysis. Neurol Sci 2013; 34; 819-830.

29. Mulleners W.M., Chronicle E.P., Palmer J.E. et al. Visual Cortex Excitability in Migraine with and without aura. Headache: The Journal of Head and Face pain 2001; 41(6): 565-572.

30. Gunaydin S., Soysal A., Turan A. et al. Motor and Occipital Cortex Excitability in Migraine Patients. Can J Neurol Sci 2006; 33: 63-67. 